\section{A comment on Day and Jory's note on a second stage in the formation of illusory contours}

\author{
C. R. ROWBURY \\ Artificial Intelligence Laboratory \\ Queen Mary College, University of London \\ London, E1 4NS, England
}

The role of minimally indicated boundaries has recently been highlighted as a necessary second stage in the formation of illusory contours (Day \& Jory, 1980). Their claim is that a region of enhanced brightness, once formed, spreads out to fill the shape outlined by small features such as line-ends and points. Moreover, Day and Jory suggest that "shapes with corners are fully resolved by fewer boundary markers when these indicate the corners than when there are no such abrupt changes in boundary direction. Continuous change in direction of the boundary ... would seem to require relatively more markers for resolution and illusory contour formation." This seems a reasonable suggestion given that the visual system is geared up to exploit redundancy by detecting changes (in, for example, luminance, depth, reflectance, and texture). An economic coding method is to record just the sharp changes together with the value of some measure which is assumed to be constant (or, at least, continuous) between such changes. Attneave (1954) uses this idea to suggest that points of locally high curvature (in particular, "vertices"-i.e., discontinuities in boundary direction) are very important when describing or encoding two-dimensional shapes. Thus, a particular shape can be adequately specified by a series of points representing places at which the boundary undergoes a sharp change in direction (Figure 1).

The situation becomes more complex when line-ends are used as boundary indicators, since these have an associated direction (and, hence, carry more information than points) which affects the local shape of

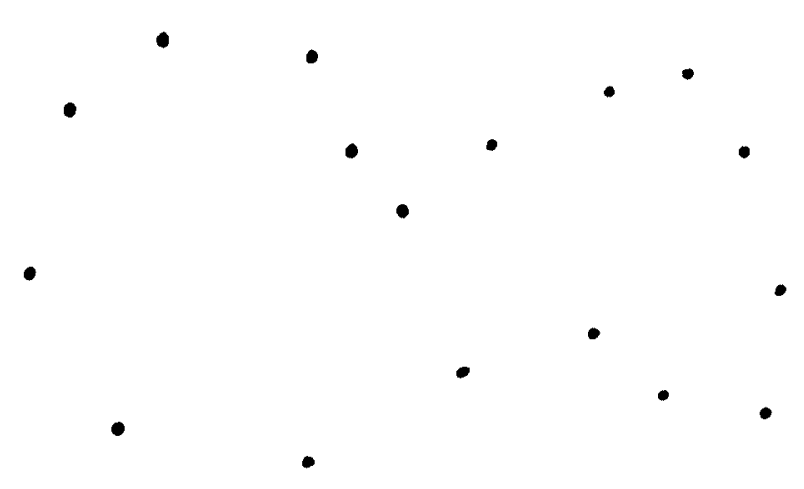

Figure 1. A figure's outline can be indicated by dots placed at points where the boundary undergoes a sharp change in direction.

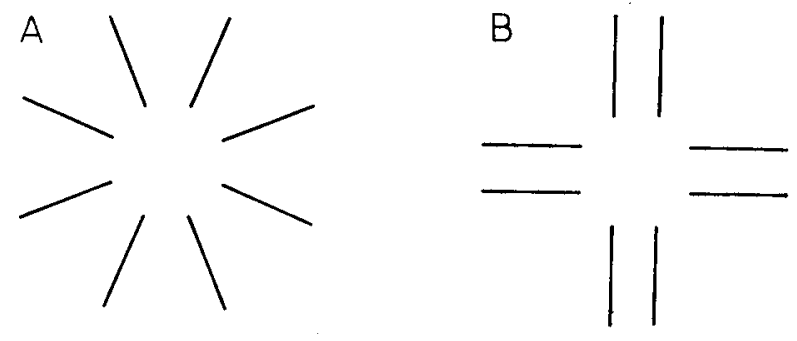

Figure 2. A change in orientation of line ends can affect the shape of an illusory contour, even though their location remains unchanged. (Figures from Kennedy, 1975.)
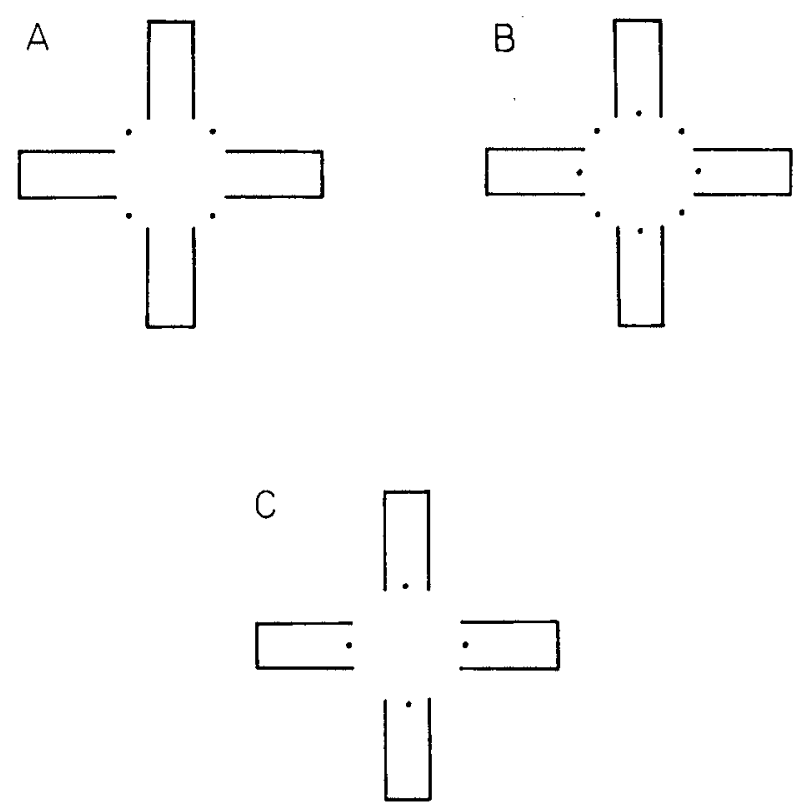

Figure 3. Illusory contours defined by minimally indicated boundaries. It is not just the number of dots which is important, but also their location. (Figure 3C from Sambin, 1974.)

any boundary passing through them. For example, compare Figures 2A and 2B (from Kennedy, 1975). Although, in each figure, the lines terminate at identical places, the resulting illusory contour takes on a different shape in each case. Kennedy (1978) has suggested that there is a preference for illusory contours that pass through line-ends at right angles, since illusory brightness (and, hence, strength or clarity of illusory contour) decreases as inducing lines veer to become tangents. It might be supposed, then, that in Figure $3 \mathrm{~A}$ there is a conflict between the square shape suggested by the line-ends, and the points, which lie on a circle. It is only when more evidence for a circular contour is added (Figure 3B) that this conflict is resolved. It is not simply a case, as Day and Jory pro- 


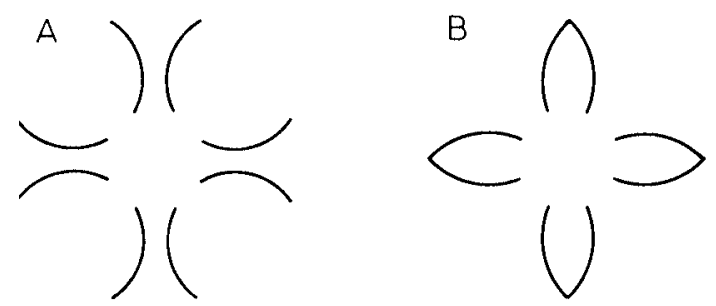

Figure 4. Grouping of lines is not sufficient alone to account for differences in illusory contour shape. (Figure 4A from Kennedy, 1975.)

pose, of continuous shapes' requiring relatively more markers for resolution, since a circular illusory contour is clearly seen in Figure 3C (from Sambin, 1974), which has the same number (and type) of markers as Figure 3A.

Kennedy (1975) argues that it is not so much the orientation of line-ends, but their possible groupings (orientation being only one of several possible grouping criteria) which determine the overall shape of an illusory contour. Kennedy presents Figure 4A in support of his argument. As in Figure 2A, the lines terminate at eight concentric loci, but Figure 4A gives rise to the same shape as Figure 2B, "presumably," says Kennedy, "because both figures involve grouping factors that act to connect pairs of similar lines." But consider Figure 4B. Again, the lines terminate at identical loci, and there is also an obvious grouping into pairs of lines similar to that in Figures $2 \mathrm{~B}$ and $4 \mathrm{~A}$, but the perceived shape is the same as that in Figure 2A. It seems, therefore, that the most important factor is the local orientations of the line ends (which are the same in Figures $2 \mathrm{~A}$ and $4 \mathrm{~B}$ ), independent of global grouping of the lines.

Hence, the proposal is that the shape of an illusory contour induced by line-ends and points is determined foremost by the local orientations of the line-ends and mediated by the relative positions of the points. If there is any ambiguity or conflict between alternative possible shapes, or if there are insufficient constraints to determine a precise shape, then the illusory contour enclosing a region of enhanced brightness appears somewhat indefinite, occasionally fluctuating between several alternative shapes in a manner reminiscent of Necker cube reversal. This proposal forms part of an overall theory of illusory contours which will be presented in full in a forthcoming paper.

In summary, whether or not there are two stages in the formation of illusory contours, the shape of such contours is that of the boundary indicated by the features which induce the contour (in this case, line-ends and points). The shape of the boundary is constrained by the local orientations of the line-ends and the fact that it must pass through both the line-ends and the points. Sometimes there are insufficient constraints to uniquely determine a precise shape, in which case the illusory contour appears somewhat indefinite.

\section{REFERENCES}

Attneave, F. Some informational aspects of visual perception. Psychological Review, 1954, 61, 183-193.

DAy, R. H., \& JoRY, M. K. A note on a second stage in the formation of illusory contours. Perception \& Psychophysics, 1980, 27, 89-91.

KenNedY, J. M. Depth at an edge, coplanarity, slant depth, change in direction and change in brightness in the production of subjective contours. Italian Journal of Psychology, 1975, 2, 107-123.

Kennedy, J. M. Illusory contours and the ends of lines. Perception, 1978, 7, 605-607.

SAmBin, M. Angular margins without gradient. Italian Journal of Psychology, 1974, 1, 355-361.

(Manuscript received November 19, 1981; accepted for publication December 8,1981 .) 